HNO 2010 • 58:747-747

DOI 10.1007/s00106-010-2153-3

Online publiziert: 4. August 2010

(c) Springer-Verlag 2010

\author{
D. Eßer \\ HNO-Klinik, Helios Klinikum Erfurt
}

\title{
Onkologische Nachsorge
}

Die Meinungen über eine sinnvolle Nachsorge von Kopf-Hals-Tumoren gehen sehr weit auseinander. Einserseits gibt es Befürworter einer sehr ausgedehnten bildgebenden Diagnostik (MRT/CT/PET/ Oberbauchsonographie, Knochenszintigraphie) in regelmäßigen kurzen Intervallen, regelmäßiger endoskopischer Kontrollen sowohl des HNO-Gebiets als auch der Endoskopie von Trachea, Bronchien, Ösophagus und Magen, der Bestimmung von Tumormarkern und spezieller symptomorientierter Untersuchungen. Andererseits gehen die Vorschläge bis zu eher unregelmäßigen Nachuntersuchungen, insbesondere zur Vermeidung des psychischen Drucks auf die Patienten, da bei einem möglichen lokoregionären Rezidiv oder einem Zweittumor die therapeutischen Möglichkeiten sehr eingeschränkt sind.

\section{(2) Die Meinungen über eine sinnvolle Nachsorge gehen sehr weit auseinander}

Ziel des vorliegenden Themenhefts ist es, unter dem Aspekt der sich ständig verbessernden und erweiternden bildgebenden Diagnostik sowie der neuen therapeutischen Optionen die Frage nach Sinn und Ausmaß der onkologischen Nachsorge neu zu stellen.

Ich möchte Ihnen mit meinen Mitarbeitern unseren Algorithmus der Nachsorge an einem großen onkologischen Krankengut aufzeigen.

Mit Herrn Dr. Aschenbach als leitendem Oberarzt des Instituts für bildgebende Diagnostik, interventionelle Radiologie und Neuroradiologie am Helios Klinikum Erfurt konnte ein ausgewiesener Fachmann auf dem Gebiet der Schnittbilddiagnostik im Kopf-Hals-Bereich gewonnen werden.
Nicht zuletzt durch die Einbeziehung der Leipziger HNO-Klinik als eines der onkologischen Zentren für die Behandlung von Kopf-Hals-Tumoren mit der Darstellung neuer therapeutischer Möglichkeiten bei der Behandlung von lokoregionären Rezidiven hoffen wir, Ihnen als interessierte Leser, aktuelle Aspekte des onkologischen Dispensaire vorstellen zu können.

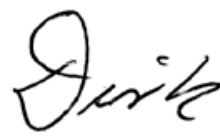

D. Eßer

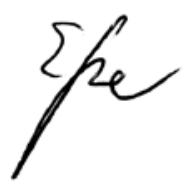

\section{Korrespondenzadresse Prof. Dr. D. Eßer}

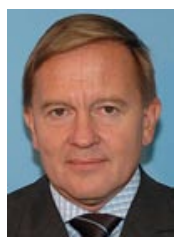

HNO-Klinik, Helios Klinikum Erfurt Nordhäuser Straße 74, 99089 Erfurt dirk.esser@helios-kliniken.de 


\section{Hier steht eine Anzeige.}

Springer 